\title{
Comparison of Haemoglobin Level in Vegetarian and Non-Vegetarian Subjects
}

\author{
Dr. Dipti V. Thakker ${ }^{1}$, Harsh Shah ${ }^{2 *}$
}

${ }^{1} \mathrm{PhD}$ Student, Gujarat University, Associate Professor, Physiology Department, PDU Government Medical College, Civil hospital campus, Rajkot, Gujarat, India

${ }^{2}$ MBBS Student, PDU Government Medical College, Civil hospital campus, Rajkot, Gujarat, India

DOI: $\underline{10.36348 / \text { sijap.2019.v02i11.005 }}$

| Received: 08.11.2019| Accepted: 15.11.2019| Published: 24.11.2019

*Corresponding author: Harsh Shah

\section{Abstract}

Dietary habits are an important determinant of health. In vegetarian diet meat (including fowl) or seafood, or products containing those foods are not included. Current recommendations for all the nutrients can be fulfilled by the vegetarian diet. Sometimes to achieve proper amount of important nutrients, supplements or fortified foods can be added in the diet. Researchers reported that vegetarian diets are beneficial in the prevention and treatment of certain diseases, such as cardiovascular disease, hypertension, diabetes, cancer, osteoporosis, renal disease and dementia, as well as diverticular disease, gallstones and rheumatoid arthritis. In this study we compared the haemoglobin level in vegetarian and nonvegetarian population from Rajkot city, Gujarat state. About 100 individuals of the age group of 16-50 years participated in the present study. Based on the dietary history, participants were divided into two groups as vegetarians and nonvegetarians. We conclude that in our study there is no significant difference in Haemoglobin concentration between two study groups. This shows that non-vegetarian diet does not have any beneficiary effect on haematological parameters in our study group.

Keywords: Dietary habits, Vegetarian, seafood, hypertension.

Copyright @ 2019: This is an open-access article distributed under the terms of the Creative Commons Attribution license which permits unrestricted use, distribution, and reproduction in any medium for non-commercial use (NonCommercial, or CC-BY-NC) provided the original author and source are credited.

\section{INTRODUCTION}

According to current guidelines, a healthy dietary pattern is high in vegetables, fruit, whole grains, seafood, legumes, and nuts, and includes a modest amount of low- and non-fat dairy products. It is also low in red and processed meat, sugar-sweetened foods and beverages, and refined grains [1].

Vegetarian diets, when properly planned, like all diets, should promote proper growth and development and can be adopted during all phases of life [2].

Many studies have demonstrated the
association between meat consumption and
hypertension, risk of heart disease, metabolic disorders,
and mortality [3] and the protective effect of a
vegetarian diet, associated with a healthy lifestyle and
the maintenance of adequate body weight, offering
some protection against these chronic diseases [4].

It was proven by the review that vegetarian diet contains all the nutrients in adequate amount to fulfil nutritional requirement of pregnant female and also of the infants. Vegetarian diet is also associated with decreased risk of death from ischemic heart diseases. The levels of low-density lipoprotein cholesterol levels, blood pressure, and blood sugar are lower in vegetarian group than nonvegetarians. Also, vegetarian diet is associated with less body mass index and lower overall cancer rates.

In the randomised Dietary Approach to Adults with Systolic Hypertension (DASH) study, this dietary pattern was shown to reduce blood pressure [5], and insulin resistance [6].

More recently the Prospective Urban Rural Epidemiological (PURE) study reported that a diet which included more dairy food and meat is associated with lower all cause and cardiovascular mortality in 138,000 people from lower, middle- and high-income countries. This large study raises questions on whether and how dairy food and meat consumption may influence mortality risk, including their impact on known cardio-metabolic risk factors. 
Vegetarian diet consists of less intake of saturated fat, cholesterol and animal protein and higher intakes of complex carbohydrates, dietary fibres, magnesium, folic acid, vitamin $\mathrm{C}$ and $\mathrm{E}$, carotenoids and other phytochemicals. If the vegetarian diet is balanced, it is appropriate for all the stages of life cycle, including children, adolescents, pregnant and lactating women, the elderly and competitive athletes.

However, other studies indicate risks and health hazards when a vegetarian diet is unbalanced or too restrictive in foods derived from animals or even vegetables. The risks include the incidence of iron deficiency anaemia and megaloblastic anaemia, caused by iron and cobalamin (vitamin $\mathrm{B}_{12}$ ) deficiency, respectively [7].

The present study was undertaken to observe Haemoglobin concentration in vegetarians and nonvegetarians and to create awareness among the general population.

In vegetarian individuals also iron intake is reported not to be less than non-vegetarian, and a study of Australian women $(n=74)$ showed that vegetarians were not more likely to be iron deficient than nonvegetarians [8]. However, in several studies, means reported for both vegetarian and non-vegetarian women did not meet recommended amounts for premenopausal women [9].

Furthermore, the Institute of Medicine (IOM) has recommended that vegetarians consume $80 \%$ more than the RDA for iron due to the lower bioavailability of the nonheme iron in vegetarian diets [10].

The iron in plant foods is nonheme iron, which is sensitive to both inhibitors and enhancers of iron absorption. Inhibitors of iron absorption include phytates, calcium, and the polyphenolics in tea, coffee, herb teas, and cocoa. Fibres only slightly inhibits iron absorption. Phytate level can be decreased by some food preparation techniques such as soaking and sprouting beans, grains, and seeds, and the leavening of bread, and thereby enhance iron absorption.

Other fermentation processes, such as those used to make miso and tempeh, may also improve iron bioavailability.

The vitamin B-12 status of some vegetarians is less than adequate due to not regularly consuming reliable sources of vitamin B-12. Lacto-ovo-vegetarians can obtain adequate vitamin B-12 from dairy foods, eggs, or other reliable vitamin B-12 sources (fortified foods and supplements), if regularly consumed. For vegans, vitamin B-12 must be obtained from regular use of vitamin B-12-fortified foods, such as fortified soy and rice beverages, some breakfast cereals and meat analogues, or Red Star Vegetarian Support Formula nutritional yeast. In absence of these, daily vitamin B12 supplement is needed. Significant amount of active vitamin B12 is not present in unfortified plant food.

Haematological symptoms of vitamin B12 deficiency are masked by rich folacin present in vegetarian diets. So that vitamin B-12 deficiency may go undetected until after neurological signs and symptoms may be manifest.

\section{AIM AND OBJECTIVES}

This study aims to assess the haemoglobin level in vegetarian and non-vegetarian population from Rajkot city, Gujarat state.

\section{MATERIALS AND METHODS}

About 100 individuals of the age group of 1650 years participated in the present study. They were hospital staff including doctors, nurses, medical assistants and attendants. History of the subject including age, sex, height and weight were recorded. Subjects were divided into 2 groups according to their dietary patterns- a) Non-vegetarians b) Vegetarians Subjects were classified as nonvegetarians if foods of plant and animal origin, including meat, fowl, eggs, milk and other dairy products, and fish were included in their diet; Vegetarians if foods of plant and dairy products were included in their diet.

Study protocol was approved by Institutional Ethics Committee of PDU Government Medical College, Rajkot. The study subjects were briefed about the procedure and informed consent was obtained from each participant.

Haematological tests carried out were $\mathrm{Hb}$ estimation. The data were analysed $\mathrm{Z}$ test for two samples and $\mathrm{P}$ value $<0.05$ was considered as significant.

\section{OBSERVATION AND RESULTS}

In the present study out of the total 100 subjects recruited, a group of 50 were vegetarians and 50 were non-vegetarians. BMI, Heart rate, Systolic Blood Pressure, Diastolic blood pressure, Haemoglobin, RBC count and PCV are compared between vegetarian and non-vegetarian subjects. 


\section{Correlation between Diet and Haemoglobin}

Table-1: Haemoglobin range in Non-vegetarian subjects

\begin{tabular}{|c|c|}
\hline Haemoglobin $(\mathbf{g m} / \mathbf{d l})$ & Number of subjects (Non-Vegetarian) \\
\hline $9-11$ & 9 \\
\hline $11-13$ & 7 \\
\hline $13-15$ & 23 \\
\hline $15-17$ & 11 \\
\hline
\end{tabular}

Table-2: Haemoglobin range in Vegetarian subjects

\begin{tabular}{|c|c|}
\hline Haemoglobin $\mathbf{( g m / d l )}$ & Number of subjects (Vegetarian) \\
\hline $9-11$ & 6 \\
\hline $11-13$ & 13 \\
\hline $13-15$ & 24 \\
\hline $15-17$ & 7 \\
\hline
\end{tabular}

Table-3: Comparison of values in Non-vegetarian and vegetarian subjects

\begin{tabular}{|l|l|l|}
\hline Haemoglobin (Non-Vegetarian) & Mean: 13.44 & p Value: 0.9 \\
\cline { 2 - 2 } & SD: 2.02 & \multirow{2}{*}{ Not significant } \\
\hline Haemoglobin (Vegetarian) & Mean: 13.46 & \\
\cline { 2 - 2 } & SD: 1.58 & \\
\hline
\end{tabular}

\section{Two sample z-test}
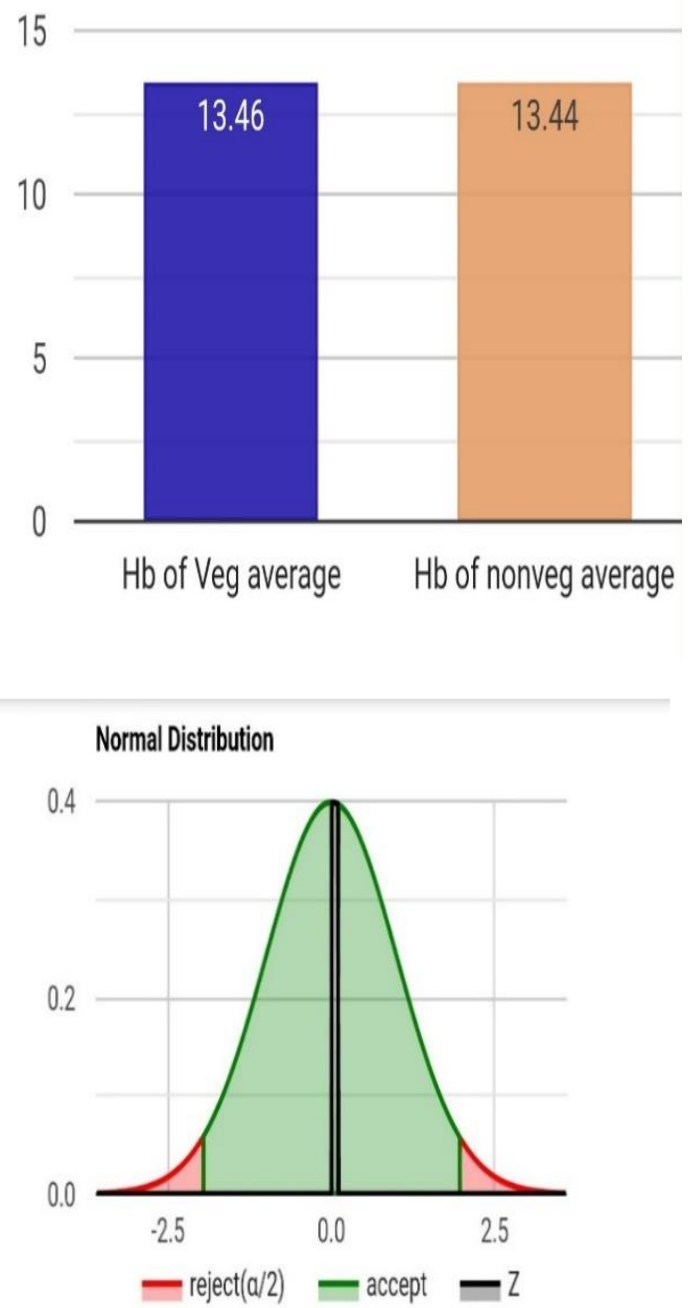

Average Haemoglobin concentration of vegetarian population is considered to be statistically equivalent to average of Haemoglobin concentration of nonvegetarian population. The difference between average of Haemoglobin concentration of nonvegetarian and vegetarian population is not large enough to be statistically significant. So, in this group diet has no significant effect on Haemoglobin concentration of two population groups.

\section{DISCUSSION}

It is well known that a vegetarian or vegan diet, when sensibly managed, can make a contribution to the prevention and therapy of illnesses in all phases of life. More and more people are thus adopting a vegetarian or vegan lifestyle [11].

As Vitamin $B_{12}$ is absent in plant foods; diets based entirely on plant foods are devoid of the vitamin unless they are supplemented or contaminated. In healthy young and middle-aged individuals who adopt vegan diets, diminution of vitamin $\mathrm{B}_{12}$ stores is thought to be rare, even if no dietary source is consumed, depletion of vitamin $B_{12}$ level requires several years to take place.

Deficiencies in some nutrients such as vitamin

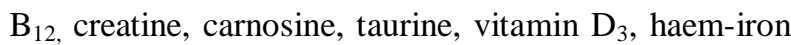
and the omega-3 fatty acids may also influence cardiovascular health [12].

Vegetarians may have nutritional inadequacy for vitamin $\mathrm{B}_{12}$, protein, and calcium compared to nonvegetarians. Hence, the potential adverse effects of vegetarian diets should be considered. In the EPICOxford study, vegans had $30 \%$ higher fracture rates than meat-eaters. Vegetarians had no longer higher 
fracture rate, when adjusted for calcium intake. In a review, Dunham et al., stated that as compared with other vegetarians, vegans have higher rates of vitamin $\mathrm{B}_{12}$ deficiency [13].

One of the studies showed that Five-year lactoovo-vegetarian diet did not result in the risk of vitamin B12 deficiency in healthy subjects [14].

\section{CONCLUSION}

Appropriately planned vegetarian diets have been shown to be healthful, nutritionally adequate, and may be beneficial in the prevention and treatment of certain diseases. For all the stages of life cycle vegetarian diets are appropriate.

We conclude that in our study there is no significant difference in Haemoglobin concentration between two study groups. This shows that nonvegetarian diet does not have any beneficiary effect on haematological parameters in our study group.

\section{ACKNOWLEDGEMENT}

The authors are grateful to all groups of Vegetarian and Non-vegetarian subjects of Rajkot city, Gujarat state who participated in the study. Our thanks are also for the Pathology Department for their support to carry out haematological investigation. Our special thanks to Dean madam, Dr. Gauravi Dhruva and Physiology Department HOD, Dr. R S. Trivedi for their kind support throughout the study.

\section{REFERENCES}

1. Dietary Guidelines for Americans. United States of America: USDA Center for Nutrition Policy and Promotion. 2015-2020.

2. Slywitch, E. (2012). Guide feeding vegetarian diets for adults. São Paulo: Sociedade Brasileira Vegetariana.

3. Acosta-Navarro, J. C., Prado, S. M. C., Sanchez E. T., Escobedo, D., \& Pineda, Z. (1998). Blood pressure, lipid profile and other biochemical parameters among vegetarians, semi-vegetarians and omnivores Peruvians. The Lima Study. An Paul Med Cir, 125: 87-101.

4. McEvoy, C. T., Temple, N., \& Woodside, J. V. (2012). Vegetarian diets, low-meat diets and health: a review. Public health nutrition, 15(12), 2287-2294.
5. Appel, L. J., Moore, T. J., Obarzanek, E., Vollmer, W. M., Svetkey, L. P., Sacks, F. M., ... \& Lin, P. H. (1997). A clinical trial of the effects of dietary patterns on blood pressure. New England journal of medicine, 336(16), 1117-1124.

6. Liese, A. D., Nichols, M., Sun, X., D'Agostino, R. B., \& Haffner, S. M. (2009). Adherence to the DASH Diet is inversely associated with incidence of type 2 diabetes: the insulin resistance atherosclerosis study. Diabetes care, 32(8), 14341436.

7. Campos, F. A. A. C., Cheavegatti, D., Martins, F. A., \& Seixas, L. A. (2011). Enfermeiros no cuidado da saúde de vegetarianos; 63 CBEn; 3 a 6 de outubro de 2011; Macéo. Brasília: Aben.

8. Ball, M. J., \& Bartlett, M. A. (1999). Dietary intake and iron status of Australian vegetarian women. The American journal of clinical nutrition, 70(3), 353-358.

9. Haddad, E. H., \& Tanzman, J. S. (2003). What do vegetarians in the United States eat?. The American journal of clinical nutrition, 78(3), 626S-632S.

10. Russell, R. M., Beard, J. L., Cousins, R. J., Dunn, J. T., Ferland, G., Hambidge, K. M., ... \& Suttie, J. W. (2001). Dietary reference intakes for vitamin A, vitamin $\mathrm{K}$, arsenic, boron, chromium, copper, iodine, iron, manganese, molybdenum, nickel, silicon, vanadium, and zinc. A Report of the Panel on Micronutrients, Subcommittees on Upper Reference Levels of Nutrients and of Interpretation and Uses of Dietary Reference Intakes, and the Standing Committee on the Scientific Evaluation of Dietary Reference Intakes Food and Nutrition Board Institute of Medicine.

11. Fuhrman, J., \& Ferreri, D. M. (2010). Fueling the vegetarian (vegan) athlete. Current sports medicine reports, 9(4), 233-241.

12. Craig, W. J. (2009). Health effects of vegan diets. The American journal of clinical nutrition, 89(5), 1627S-1633S.

13. Dunham, L., \& Kollar, L. M. (2006). Vegetarian eating for children and adolescents. Journal of Pediatric Health Care, 20(1), 27-34.

14. Mądry, E., Lisowska, A., Chabasińska, M., Przysławski, J., Schlegel-Zawadzka, M., \& Walkowiak, J. (2009). Effect of lacto-ovovegetarian diet on serum vitamin B12 concentrations-five-year prospective study. Acta Scientiarum Polonorum Technologia Alimentaria, 8(4), 71-76. 